\title{
A NEW CARTAN-TYPE PROPERTY AND STRICT QUASICOVERINGS WHEN $p=1$ IN METRIC SPACES
}

\author{
Panu Lahti \\ University of Jyväskylä, Department of Mathematics and Statistics \\ P.O. Box 35, FI-40014 University of Jyväskylä, Finland; panu.k.lahti@jyu.fi
}

\begin{abstract}
In a complete metric space that is equipped with a doubling measure and supports a Poincaré inequality, we prove a new Cartan-type property for the fine topology in the case $p=$ 1. Then we use this property to prove the existence of 1-finely open strict subsets and strict quasicoverings of 1-finely open sets. As an application, we study fine Newton-Sobolev spaces in the case $p=1$, that is, Newton-Sobolev spaces defined on 1-finely open sets.
\end{abstract}

\section{Introduction}

Nonlinear fine potential theory in metric spaces has been studied in several papers in recent years, see [7, 8, 6]. Much of nonlinear potential theory, for $1<p<$ $\infty$, deals with $p$-harmonic functions, which are local minimizers of the $L^{p}$-norm of $|\nabla u|$. Such minimizers can be defined also in metric measure spaces by using upper gradients, and the notion can be extended to the case $p=1$ by considering functions of least gradient, which are BV functions that minimize the total variation locally; see Section 2 for definitions.

Nonlinear fine potential theory is concerned with studying $p$-harmonic functions and related superminimizers by means of the $p$-fine topology. For nonlinear fine potential theory and its history in the Euclidean setting, for $1<p<\infty$, see especially the monographs $[1,15,23]$, as well as the monograph [3] in the metric setting. The typical assumptions of a metric space, which we make also in this paper, are that the space is complete, equipped with a doubling measure, and supports a Poincaré inequality.

A central result in fine potential theory is the (weak) Cartan property for superminimizer functions. In [21] we proved the following formulation of this property in the case $p=1$.

Theorem 1.1. [21, Theorem 1.1] Let $A \subset X$ and let $x \in X \backslash A$ such that $A$ is 1-thin at $x$. Then there exist $R>0$ and $u_{1}, u_{2} \in \mathrm{BV}(X)$ that are 1-superminimizers in $B(x, R)$ such that $\max \left\{u_{1}^{\wedge}, u_{2}^{\wedge}\right\}=1$ in $A \cap B(x, R)$ and $u_{1}^{\vee}(x)=0=u_{2}^{\vee}(x)$.

In [22] we used this property to prove the so-called Choquet property concerning finely open and quasiopen sets in the case $p=1$, similarly as can be done when $1<p<\infty$ (see [8]). On the other hand, it is natural to consider an alternative version of the weak Cartan property. In the case $p>1$, superminimizers are Newton-Sobolev functions, but in the case $p=1$ they are only BV functions and so the question arises whether the functions $u_{1}, u_{2}$ above can be replaced by a Newton-Sobolev function

https://doi.org/10.5186/aasfm.2018.4364

2010 Mathematics Subject Classification: Primary 30L99, 31E05, $26 B 30$.

Key words: Metric measure space, function of bounded variation, fine topology, Cartan property, strict quasicovering, fine Newton-Sobolev space. 
(even though it would no longer be a superminimizer). In Theorem 3.11 we show that such a new Cartan-type property indeed holds.

It is said that a set $A$ is a $p$-strict subset of a set $D$ if there exists a NewtonSobolev function $u \in N^{1, p}(X)$ such that $u=1$ on $A$ and $u=0$ on $X \backslash D$. In [7] it was shown that if $U$ is a $p$-finely open set $(1<p<\infty)$ and $x \in U$, then there exists a $p$-finely open strict subset $V \Subset U$ such that $x \in V$. The proof was based on the weak Cartan property. In Theorem 4.3 we show that the analogous result is true in the case $p=1$. Here we need the Cartan-type property involving a Newton-Sobolev function (instead of the BV superminimizer functions).

This result on the existence of 1-strict subsets can be combined with the quasiLindelöf principle to prove the existence of strict quasicoverings of 1-finely open sets, that is, countable coverings by 1-finely open strict subsets. We do this in Proposition 5.4, and it is again analogous to the case $1<p<\infty$, see [7]. Such coverings will be useful in future research when considering partition of unity arguments in finely open sets. In this paper, we apply strict quasicoverings in defining and studying fine Newton-Sobolev spaces, that is, Newton-Sobolev spaces defined on finely open or quasiopen sets. In the case $1<p<\infty$, these were studied in [7]. In Section 5 we show that the theory we have developed allows us to prove directly analogous results in the case $p=1$.

\section{Preliminaries}

In this section we introduce the notation, definitions, and assumptions used in the paper. Throughout this paper, $(X, d, \mu)$ is a complete metric space that is equipped with a metric $d$ and a Borel regular outer measure $\mu$ that satisfies a doubling property, meaning that there exists a constant $C_{d} \geq 1$ such that

$$
0<\mu(B(x, 2 r)) \leq C_{d} \mu(B(x, r))<\infty
$$

for every ball $B(x, r):=\{y \in X: d(y, x)<r\}$. We also assume that $X$ supports a $(1,1)$-Poincaré inequality defined below, and that $X$ contains at least 2 points. For a ball $B=B(x, r)$ and $a>0$, we sometimes abbreviate $a B:=B(x, a r)$; note that in metric spaces, a ball (as a set) does not necessarily have a unique center and radius, but we will always understand these to be predetermined for the balls that we consider. By iterating the doubling condition, we obtain for any $x \in X$ and any $y \in B(x, R)$ with $0<r \leq R<\infty$ that

$$
\frac{\mu(B(y, r))}{\mu(B(x, R))} \geq \frac{1}{C_{d}^{2}}\left(\frac{r}{R}\right)^{Q}
$$

where $Q>1$ only depends on the doubling constant $C_{d}$. When we want to state that a constant $C$ depends on the parameters $a, b, \ldots$, we write $C=C(a, b, \ldots)$. When a property holds outside a set of $\mu$-measure zero, we say that it holds almost everywhere, abbreviated a.e.

As a complete metric space equipped with a doubling measure, $X$ is proper, that is, closed and bounded sets are compact. For any $\mu$-measurable set $D \subset X$, we define $\operatorname{Lip}_{\text {loc }}(D)$ to be the space of functions $u$ on $D$ such that for every $x \in D$ there exists $r>0$ such that $u \in \operatorname{Lip}(D \cap B(x, r))$. For an open set $\Omega \subset X$, a function $u \in \operatorname{Lip}_{\text {loc }}(\Omega)$ is then in $\operatorname{Lip}\left(\Omega^{\prime}\right)$ for every open $\Omega^{\prime} \Subset \Omega$; this notation means that $\overline{\Omega^{\prime}}$ is a compact subset of $\Omega$. Other local spaces of functions are defined analogously. 
For any $A \subset X$ and $0<R<\infty$, the restricted Hausdorff content of codimension one is defined to be

$$
\mathcal{H}_{R}(A):=\inf \left\{\sum_{i=1}^{\infty} \frac{\mu\left(B\left(x_{i}, r_{i}\right)\right)}{r_{i}}: A \subset \bigcup_{i=1}^{\infty} B\left(x_{i}, r_{i}\right), r_{i} \leq R\right\} .
$$

The codimension one Hausdorff measure of $A \subset X$ is then defined to be

$$
\mathcal{H}(A):=\lim _{R \rightarrow 0} \mathcal{H}_{R}(A)
$$

All functions defined on $X$ or its subsets will take values in $[-\infty, \infty]$. By a curve we mean a nonconstant rectifiable continuous mapping from a compact interval of the real line into $X$. A nonnegative Borel function $g$ on $X$ is an upper gradient of a function $u$ on $X$ if for all curves $\gamma$, we have

$$
|u(x)-u(y)| \leq \int_{\gamma} g d s,
$$

where $x$ and $y$ are the end points of $\gamma$ and the curve integral is defined by using an arc-length parametrization, see [16, Section 2] where upper gradients were originally introduced. We interpret $|u(x)-u(y)|=\infty$ whenever at least one of $|u(x)|,|u(y)|$ is infinite.

Let $1 \leq p<\infty$; we are going to work solely with $p=1$, but we give definitions that cover all values of $p$ where it takes no extra work. We say that a family of curves $\Gamma$ is of zero $p$-modulus if there is a nonnegative Borel function $\rho \in L^{p}(X)$ such that for all curves $\gamma \in \Gamma$, the curve integral $\int_{\gamma} \rho d s$ is infinite. A property is said to hold for $p$-almost every curve if it fails only for a curve family with zero $p$-modulus. If $g$ is a nonnegative $\mu$-measurable function on $X$ and (2.2) holds for $p$-almost every curve, we say that $g$ is a $p$-weak upper gradient of $u$. By only considering curves $\gamma$ in a set $D \subset X$, we can talk about a function $g$ being a ( $p$-weak) upper gradient of $u$ in $D$.

Let $D \subset X$ be a $\mu$-measurable set. We define the norm

$$
\|u\|_{N^{1, p}(D)}:=\|u\|_{L^{p}(D)}+\inf \|g\|_{L^{p}(D)}
$$

where the infimum is taken over all $p$-weak upper gradients $g$ of $u$ in $D$. The usual Sobolev space $W^{1, p}$ is replaced in the metric setting by the Newton-Sobolev space

$$
N^{1, p}(D):=\left\{u:\|u\|_{N^{1, p}(D)}<\infty\right\}
$$

which was first introduced in [25]. We understand every Newton-Sobolev function to be defined at every $x \in D$ (even though $\|\cdot\|_{N^{1, p}(D)}$ is then only a seminorm). It is known that for any $u \in N_{\text {loc }}^{1, p}(D)$, there exists a minimal $p$-weak upper gradient of $u$ in $D$, always denoted by $g_{u}$, satisfying $g_{u} \leq g$ a.e. on $D$ for any $p$-weak upper gradient $g \in L_{\mathrm{loc}}^{p}(D)$ of $u$ in $D$, see [3, Theorem 2.25].

For any $D \subset X$, the space of Newton-Sobolev functions with zero boundary values is defined to be

$$
N_{0}^{1, p}(D):=\left\{\left.u\right|_{D}: u \in N^{1, p}(X) \text { and } u=0 \text { on } X \backslash D\right\} .
$$

This is a subspace of $N^{1, p}(D)$ when $D$ is $\mu$-measurable, and it can always be understood to be a subspace of $N^{1, p}(X)$.

The $p$-capacity of a set $A \subset X$ is

$$
\operatorname{Cap}_{p}(A):=\inf \|u\|_{N^{1, p}(X)}
$$


where the infimum is taken over all functions $u \in N^{1, p}(X)$ such that $u \geq 1$ on $A$. If a property holds outside a set $A \subset X$ with $\operatorname{Cap}_{p}(A)=0$, we say that it holds $p$-quasieverywhere, or $p$-q.e. If $D \subset X$ is $\mu$-measurable, then

$$
\|u\|_{N^{1, p}(D)}=0 \quad \text { if } \quad u=0 \text {-q.e. on } D,
$$

see [3, Proposition 1.61].

We know that $\mathrm{Cap}_{p}$ is an outer capacity, meaning that

$$
\operatorname{Cap}_{p}(A)=\inf _{\substack{W \text { open } \\ A \subset W}} \operatorname{Cap}_{p}(W)
$$

for any $A \subset X$, see e.g. [3, Theorem 5.31]. By [14, Theorem 4.3, Theorem 5.1], for any $A \subset X$ it holds that

$$
\operatorname{Cap}_{1}(A)=0 \text { if and only if } \mathcal{H}(A)=0 .
$$

We say that a set $U \subset X$ is $p$-quasiopen if for every $\varepsilon>0$ there is an open set $G \subset X$ such that $\operatorname{Cap}_{p}(G)<\varepsilon$ and $U \cup G$ is open. We say that a function $u$ defined on a set $D \subset X$ is $p$-quasicontinuous on $D$ if for every $\varepsilon>0$ there is an open set $G \subset X$ such that $\operatorname{Cap}_{p}(G)<\varepsilon$ and $\left.u\right|_{D \backslash G}$ is continuous (as a real-valued function). It is a well-known fact that Newton-Sobolev functions are quasicontinuous; for a proof of the following theorem, see [10, Theorem 1.1] or [3, Theorem 5.29].

Theorem 2.5. Let $\Omega \subset X$ be open and let $u \in N^{1, p}(\Omega)$ (with $1 \leq p<\infty$ ). Then $u$ is $p$-quasicontinuous on $\Omega$.

The variational $p$-capacity of a set $A \subset D$ with respect to $D \subset X$ is given by

$$
\operatorname{cap}_{p}(A, D):=\inf \int_{X} g_{u}^{p} d \mu
$$

where the infimum is taken over functions $u \in N_{0}^{1, p}(D)$ such that $u \geq 1$ on $A$, and $g_{u}$ is the minimal $p$-weak upper gradient of $u$ (in $X$ ). By truncation, we see that we can also assume that $0 \leq u \leq 1$ on $X$ (and the same applies to the $p$-capacity). For basic properties satisfied by capacities, such as monotonicity and countable subadditivity, see $[3,4]$.

Next we recall the definition and basic properties of functions of bounded variation on metric spaces, following [24]. See also the monographs $[2,11,12,13,26]$ for the classical theory in the Euclidean setting. Let $\Omega \subset X$ be an open set. Given $u \in L_{\text {loc }}^{1}(\Omega)$, the total variation of $u$ in $\Omega$ is defined to be

$$
\|D u\|(\Omega):=\inf \left\{\liminf _{i \rightarrow \infty} \int_{\Omega} g_{u_{i}} d \mu: u_{i} \in \operatorname{Lip}_{\mathrm{loc}}(\Omega), u_{i} \rightarrow u \text { in } L_{\mathrm{loc}}^{1}(\Omega)\right\},
$$

where each $g_{u_{i}}$ is the minimal 1-weak upper gradient of $u_{i}$ in $\Omega$. (In [24], local Lipschitz constants were used instead of upper gradients, but the properties of the total variation can be proved similarly with either definition.) We say that a function $u \in L^{1}(\Omega)$ is of bounded variation, and denote $u \in \mathrm{BV}(\Omega)$, if $\|D u\|(\Omega)<\infty$. For an arbitrary set $A \subset X$, we define

$$
\|D u\|(A):=\inf _{\substack{W \text { open } \\ A \subset W}}\|D u\|(W) .
$$

If $u \in L_{\text {loc }}^{1}(\Omega)$ and $\|D u\|(\Omega)<\infty$, then $\|D u\|(\cdot)$ is a Radon measure on $\Omega$ by [24, Theorem 3.4]. A $\mu$-measurable set $E \subset X$ is said to be of finite perimeter if $\left\|D \chi_{E}\right\|(X)<\infty$, where $\chi_{E}$ is the characteristic function of $E$. The perimeter of $E$ in $\Omega$ is also denoted by $P(E, \Omega):=\left\|D \chi_{E}\right\|(\Omega)$. 
The lower and upper approximate limits of a function $u$ on $X$ are defined respectively by

and

$$
u^{\wedge}(x):=\sup \left\{t \in \mathbf{R}: \lim _{r \rightarrow 0} \frac{\mu(B(x, r) \cap\{u<t\})}{\mu(B(x, r))}=0\right\}
$$

$$
u^{\vee}(x):=\inf \left\{t \in \mathbf{R}: \lim _{r \rightarrow 0} \frac{\mu(B(x, r) \cap\{u>t\})}{\mu(B(x, r))}=0\right\} .
$$

Unlike Newton-Sobolev functions, we understand BV functions to be $\mu$-equivalence classes. To consider fine properties, we need to consider the pointwise representatives $u^{\wedge}$ and $u^{\vee}$.

We will assume throughout the paper that $X$ supports a $(1,1)$-Poincaré inequality, meaning that there exist constants $C_{P}>0$ and $\lambda \geq 1$ such that for every ball $B(x, r)$, every $u \in L_{\text {loc }}^{1}(X)$, and every upper gradient $g$ of $u$, we have

$$
f_{B(x, r)}\left|u-u_{B(x, r)}\right| d \mu \leq C_{P} r f_{B(x, \lambda r)} g d \mu,
$$

where

$$
u_{B(x, r)}:=f_{B(x, r)} u d \mu:=\frac{1}{\mu(B(x, r))} \int_{B(x, r)} u d \mu .
$$

The (1,1)-Poincaré inequality implies the following Sobolev inequality: if $x \in X$, $0<r<\frac{1}{4} \operatorname{diam} X$, and $u \in N_{0}^{1,1}(B(x, r))$, then

$$
\int_{B(x, r)}|u| d \mu \leq C_{S} r \int_{B(x, r)} g_{u} d \mu
$$

for some constant $C_{S}=C_{S}\left(C_{d}, C_{P}\right) \geq 1$, see [3, Theorem 5.51]. By applying this to approximating functions in the definition of the total variation, we obtain for any $x \in X, 0<r<\frac{1}{4} \operatorname{diam} X$, and any $\mu$-measurable set $E \subset B(x, r)$

$$
\mu(E) \leq C_{S} r P(E, X) .
$$

Next we define the fine topology in the case $p=1$.

Definition 2.9. We say that $A \subset X$ is 1-thin at the point $x \in X$ if

$$
\lim _{r \rightarrow 0} r \frac{\operatorname{cap}_{1}(A \cap B(x, r), B(x, 2 r))}{\mu(B(x, r))}=0 .
$$

We say that a set $U \subset X$ is 1-finely open if $X \backslash U$ is 1-thin at every $x \in U$. Then we define the 1-fine topology as the collection of 1-finely open sets on $X$ (see [20, Lemma 4.2] for a proof of the fact that this is indeed a topology).

We denote the 1-fine interior of a set $H \subset X$, i.e. the largest 1-finely open set contained in $H$, by fine-int $H$. We denote the 1-fine closure of $H \subset X$, i.e. the smallest 1-finely closed set containing $H$, by $\bar{H}^{1}$. We define the 1-base $b_{1} H$ of $H \subset X$ to be the set of points in $X$ where $H$ is not 1 -thin. We say that a function $u$ defined on a set $U \subset X$ is 1-finely continuous at $x \in U$ if it is continuous at $x$ when $U$ is equipped with the induced 1-fine topology on $U$ and $[-\infty, \infty]$ is equipped with the usual topology.

By [3, Proposition 6.16], for all $x \in X$ and $0<r<\frac{1}{8} \operatorname{diam} X$ (in fact, the second inequality holds for all $r>0$ )

$$
\frac{\mu(B(x, r))}{2 C_{S} r} \leq \operatorname{cap}_{1}(B(x, r), B(x, 2 r)) \leq \frac{C_{d} \mu(B(x, r))}{r},
$$


and so obviously $W \subset b_{1} W$ for any open set $W \subset X$.

The following fact is given in [19, Proposition 3.3]:

$$
\operatorname{Cap}_{1}\left(\bar{A}^{1}\right)=\operatorname{Cap}_{1}(A) \text { for any } A \subset X \text {. }
$$

The following result describes the close relationship between finely open and quasiopen sets.

Theorem 2.12. [22, Corollary 6.12] $A$ set $U \subset X$ is 1-quasiopen if and only if it is the union of a 1-finely open set and a $\mathcal{H}$-negligible set.

For an open set $\Omega \subset X$, we denote by $\operatorname{BV}_{c}(\Omega)$ the class of functions $\varphi \in \operatorname{BV}(\Omega)$ with compact support in $\Omega$, that is, $\operatorname{spt} \varphi \Subset \Omega$.

Definition 2.13. We say that $u \in \operatorname{BV}_{\text {loc }}(\Omega)$ is a 1 -minimizer in $\Omega$ if for all $\varphi \in \mathrm{BV}_{c}(\Omega)$

$$
\|D u\|(\operatorname{spt} \varphi) \leq\|D(u+\varphi)\|(\operatorname{spt} \varphi) .
$$

We say that $u \in \mathrm{BV}_{\text {loc }}(\Omega)$ is a 1-superminimizer in $\Omega$ if (2.14) holds for all nonnegative $\varphi \in \mathrm{BV}_{c}(\Omega)$.

More precisely, we should talk about spt $|\varphi|^{\vee}$, since $\varphi$ is only a.e. defined. In the literature, 1-minimizers are usually called functions of least gradient.

\section{A new Cartan-type property}

In this section we prove the new Cartan-type property, given in Theorem 3.11. First we take note of a few results that we will need in the proofs; the following is given in [3, Lemma 11.22].

Lemma 3.1. Let $x \in X, r>0$, and $A \subset B(x, r)$. Then for every $1<s<t$ with $t r<\frac{1}{4} \operatorname{diam} X$, we have

$$
\operatorname{cap}_{1}(A, B(x, t r)) \leq \operatorname{cap}_{1}(A, B(x, s r)) \leq C_{S}\left(1+\frac{t}{s-1}\right) \operatorname{cap}_{1}(A, B(x, t r)),
$$

where $C_{S}$ is the constant from the Sobolev inequality (2.7).

Theorem 3.2. [21, Theorem 3.11] Let $u$ be a 1-superminimizer in an open set $\Omega \subset X$. Then $u^{\wedge}: \Omega \rightarrow(-\infty, \infty]$ is lower semicontinuous.

As mentioned in the introduction, in [21] we proved a weak Cartan property for $p=1$, more precisely in the following form.

Theorem 3.3. [21, Theorem 5.2] Let $A \subset X$ and let $x \in X \backslash A$ be such that $A$ is 1-thin at $x$. Then there exist $R>0$ and $E_{0}, E_{1} \subset X$ such that $\chi_{E_{0}}, \chi_{E_{1}} \in \mathrm{BV}(X)$, $\chi_{E_{0}}$ and $\chi_{E_{1}}$ are 1-superminimizers in $B(x, R), \max \left\{\chi_{E_{0}}^{\wedge}, \chi_{E_{1}}^{\wedge}\right\}=1$ in $A \cap B(x, R)$, $\chi_{E_{0}}^{\vee}(x)=0=\chi_{E_{1}}^{\vee}(x),\left\{\max \left\{\chi_{E_{0}}^{\vee}, \chi_{E_{1}}^{\vee}\right\}>0\right\}$ is 1-thin at $x$, and

$$
\lim _{r \rightarrow 0} r \frac{P\left(E_{0}, B(x, r)\right)}{\mu(B(x, r))}=0, \quad \lim _{r \rightarrow 0} r \frac{P\left(E_{1}, B(x, r)\right)}{\mu(B(x, r))}=0 .
$$

Now we collect a few facts that are not included in the above statement, but are given in the proof in [21]. Defining $B_{j}:=B\left(x, 2^{-j} R\right)$ and $H_{j}:=B_{j} \backslash \frac{9}{10} \overline{B_{j+1}}$ for $j=0,1, \ldots$, there exists an open set $W \supset A$ that is 1 -thin at $x$,

$$
W \cap \bigcup_{j=0,2, \ldots} H_{j} \subset E_{0} \text { and } W \cap \bigcup_{j=1,3, \ldots} H_{j} \subset E_{1},
$$


and (see [21, Eq. (5.4)])

$$
\begin{aligned}
& E_{0} \subset\left(\frac{3}{2} B_{0} \backslash \frac{4}{5} B_{1}\right) \cup \bigcup_{j=2,4, \ldots}^{\infty} \frac{5}{4} B_{j} \backslash \frac{4}{5} B_{j+1} \text { and } \\
& E_{1} \subset\left(\frac{3}{2} B_{1} \backslash \frac{4}{5} B_{2}\right) \cup \bigcup_{j=3,5, \ldots}^{\infty} \frac{5}{4} B_{j} \backslash \frac{4}{5} B_{j+1} .
\end{aligned}
$$

Moreover, by [21, Eq. (5.5)], for all $i=2,4,6, \ldots$ we have

$$
P\left(E_{0} \cap \frac{5}{4} B_{i}, X\right) \leq 5 C_{S} \operatorname{cap}_{1}\left(W \cap B_{i}, 2 B_{i}\right),
$$

and similarly for all $i=3,5,7, \ldots$,

$$
P\left(E_{1} \cap \frac{5}{4} B_{i}, X\right) \leq 5 C_{S} \operatorname{cap}_{1}\left(W \cap B_{i}, 2 B_{i}\right) .
$$

From the proof it can also be seen that if $R>0$ is chosen to be smaller, all of the above results still hold. The same will then apply to the conclusion of the next lemma. Let $B_{j}$ and $H_{j}$ be defined as above.

Lemma 3.8. Let $A \subset X$ and let $x \in X \backslash A$ be such that $A$ is 1-thin at $x$. Then there exists a number $R>0$, an open set $W \supset A$ that is 1-thin at $x$, and open sets $F_{j} \supset W \cap H_{j}$ such that $F_{j} \subset \frac{5}{4} B_{j} \backslash \frac{3}{4} B_{j+1}$ for all $j=0,1, \ldots$, and

$$
\sum_{j=i}^{\infty} P\left(F_{j}, X\right) \leq 50 C_{S}^{2} \operatorname{cap}_{1}\left(W \cap B_{i}, 2 B_{i}\right)
$$

for all $i=0,1, \ldots$.

Proof. By using the weak Cartan property (Theorem 3.3), choose $R>0$ and $E_{0}, E_{1} \subset X$ such that $\chi_{E_{0}}, \chi_{E_{1}} \in \mathrm{BV}(X)$ and $\chi_{E_{0}}$ and $\chi_{E_{1}}$ are 1-superminimizers in $B(x, R)$. We can assume that $R<\frac{1}{2} \operatorname{diam} X$. Also let $W \supset A$ be an open set that is 1 -thin at $x$, as described above. Define

$$
F_{j}:=\left\{\chi_{E_{0}}^{\wedge}>0\right\} \cap \frac{5}{4} B_{j} \backslash \frac{3}{4} \overline{B_{j+1}} \quad \text { for } j=2,4, \ldots,
$$

and

$$
F_{j}:=\left\{\chi_{E_{1}}^{\wedge}>0\right\} \cap \frac{5}{4} B_{j} \backslash \frac{3}{4} \overline{B_{j+1}} \quad \text { for } j=3,5, \ldots
$$

By (3.4), we have $F_{j} \supset W \cap H_{j}$ for all $j=2,3, \ldots$ as desired. The sets $F_{j}$ are open by Theorem 3.2. By Lebesgue's differentiation theorem, the sets $\left\{\chi_{E_{0}}^{\wedge}>0\right\}$ and $\left\{\chi_{E_{1}}^{\wedge}>0\right\}$ differ from $E_{0}$ and $E_{1}$, respectively, only by a set of $\mu$-measure zero. Thus by (3.5) and the fact that the sets $F_{j}$ are at a positive distance from each other, we find that for all $i=2,4, \ldots$,

$$
P\left(E_{0} \cap \frac{5}{4} B_{i}, X\right)=P\left(\bigcup_{j=i, i+2, \ldots} F_{j}, X\right)=\sum_{j=i, i+2, \ldots} P\left(F_{j}, X\right),
$$

and similarly for all $i=3,5, \ldots$,

$$
P\left(E_{1} \cap \frac{5}{4} B_{i}, X\right)=\sum_{j=i, i+2, \ldots} P\left(F_{j}, X\right) .
$$


Combining these with (3.6) and (3.7), and using Lemma 3.1, we have for all $i=$ $2,3, \ldots$

$$
\begin{aligned}
\sum_{j=i}^{\infty} P\left(F_{j}, X\right) & \leq 5 C_{S}\left(\operatorname{cap}_{1}\left(W \cap B_{i}, 2 B_{i}\right)+\operatorname{cap}_{1}\left(W \cap B_{i+1}, 2 B_{i+1}\right)\right) \\
& \leq 5 C_{S}\left(\operatorname{cap}_{1}\left(W \cap B_{i}, 2 B_{i}\right)+5 C_{S} \operatorname{cap}_{1}\left(W \cap B_{i+1}, 4 B_{i+1}\right)\right) \\
& \leq 25 C_{S}^{2}\left(\operatorname{cap}_{1}\left(W \cap B_{i}, 2 B_{i}\right)+\operatorname{cap}_{1}\left(W \cap B_{i}, 4 B_{i+1}\right)\right) \\
& =50 C_{S}^{2} \operatorname{cap}_{1}\left(W \cap B_{i}, 2 B_{i}\right)
\end{aligned}
$$

Then by replacing $R$ with $R / 4$, we have the result.

Recall the constant $\lambda \geq 1$ from the Poincaré inequality (2.6). We have the following boxing inequality from [18, Theorem 3.1]. Note that in [18] it is assumed that $\mu(X)=\infty$, but the proof reveals that we can alternatively assume $\mu(F)<$ $\mu(X) / 2$.

Theorem 3.10. Let $F \subset X$ be an open set of finite perimeter with $\mu(F)<$ $\mu(X) / 2$ (in particular, $\mu(F)$ is finite). Then there exists a collection of balls $\left\{B_{k}=\right.$ $\left.B\left(x_{k}, r_{k}\right)\right\}_{k \in \mathbf{N}}$ such that the balls $\lambda B_{k}$ are disjoint, $F \subset \bigcup_{k=1}^{\infty} 5 \lambda B_{k}$,

$$
\frac{1}{2 C_{d}} \leq \frac{\mu\left(B_{k} \cap F\right)}{\mu\left(B_{k}\right)} \leq \frac{1}{2}
$$

for all $k \in \mathbf{N}$, and

$$
\sum_{k=1}^{\infty} \frac{\mu\left(5 \lambda B_{k}\right)}{5 \lambda r_{k}} \leq C_{B} P(F, X)
$$

for some constant $C_{B}=C_{B}\left(C_{d}, C_{P}, \lambda\right)$.

Now we can show the following Cartan-type property.

Theorem 3.11. Let $A \subset X$ and let $x \in X \backslash A$ be such that $A$ is 1-thin at $x$. Then there exists a number $R>0$, open sets $G \subset V \subset X$, and a function $\eta \in N_{0}^{1,1}(V)$ such that $A \cap B(x, R) \subset G, V$ is 1 -thin at $x, 0 \leq \eta \leq 1$ on $X, \eta=1$ on $G$, and

$$
\lim _{r \rightarrow 0} \frac{r}{\mu(B(x, r))}\|\eta\|_{N^{1,1}(B(x, r))}=0 .
$$

Proof. Take $R>0$, an open set $W \supset A$, and open sets $F_{j} \subset \frac{5}{4} B_{j} \backslash \frac{3}{4} B_{j+1}$ as given by Lemma 3.8. Let

$$
\delta:=\frac{1}{2^{8}(680 \lambda)^{Q} C_{d}^{3} C_{S}^{3}},
$$

where $Q>1$ is the exponent in (2.1). We can assume that $R \leq \min \left\{1, \frac{1}{8} \operatorname{diam} X\right\}$. Since $\mu(\{x\})=0$ (see [3, Corollary 4.3]), we can also assume $R$ to be so small that $\mu\left(\frac{5}{4} B_{0}\right)<\frac{1}{2} \mu(X)$, and so also $\mu\left(F_{j}\right)<\frac{1}{2} \mu(X)$ for all $j=0,1, \ldots$. Since $W$ is 1 -thin at $x$, we can further assume that $R$ is so small that

$$
2^{-j} R \frac{\operatorname{cap}_{1}\left(W \cap B_{j}, 2 B_{j}\right)}{\mu\left(B_{j}\right)}<\delta
$$

for all $j=0,1, \ldots$ Fix $j$. By the boxing inequality (Theorem 3.10) we find a collection of balls $\left\{B_{k}^{j}=B\left(x_{k}^{j}, r_{k}^{j}\right)\right\}_{k=1}^{\infty}$ such that the balls $\lambda B_{k}^{j}$ are disjoint, $F_{j} \subset$ $\bigcup_{k=1}^{\infty} 5 \lambda B_{k}^{j}$

$$
\frac{1}{2 C_{d}} \leq \frac{\mu\left(B_{k}^{j} \cap F_{j}\right)}{\mu\left(B_{k}^{j}\right)} \leq \frac{1}{2}
$$


for all $k \in \mathbf{N}$, and

$$
\sum_{k=1}^{\infty} \frac{\mu\left(5 \lambda B_{k}^{j}\right)}{5 \lambda r_{k}^{j}} \leq C_{B} P\left(F_{j}, X\right)
$$

Thus we have

$$
\begin{aligned}
\mu\left(B_{k}^{j}\right) & \leq 2 C_{d} \mu\left(B_{k}^{j} \cap F_{j}\right) \leq 2 C_{d} \mu\left(F_{j}\right) \\
& \leq 2^{2-j} R C_{d} C_{S} P\left(F_{j}, X\right) \text { by }(2.8) \\
& \leq 2^{8-j} R C_{d} C_{S}^{3} \operatorname{cap}_{1}\left(W \cap B_{j}, 2 B_{j}\right) \quad \text { by }(3.9) .
\end{aligned}
$$

Thus for all $k \in \mathbf{N}$,

$$
\frac{\mu\left(B_{k}^{j}\right)}{\mu\left(B_{j}\right)} \leq 2{ }^{8} C_{d} C_{S}^{3} 2^{-j} R \frac{\operatorname{cap}_{1}\left(W \cap B_{j}, 2 B_{j}\right)}{\mu\left(B_{j}\right)} \leq 2{ }^{8} C_{d} C_{S}^{3} \delta
$$

by (3.13). By (3.14) we necessarily have $F_{j} \cap B_{k}^{j} \neq \emptyset$ for all $k \in \mathbf{N}$, and so $\frac{5}{4} B_{j} \cap B_{k}^{j} \neq$ $\emptyset$. Now if $r_{k}^{j} \geq 2^{-j} R$ for some $k \in \mathbf{N}$, then $B_{j} \subset 4 B_{k}^{j}$ and so

$$
\frac{\mu\left(B_{k}^{j}\right)}{\mu\left(B_{j}\right)} \geq \frac{1}{C_{d}^{2}}
$$

contradicting (3.16) by our choice of $\delta$. Thus $r_{k}^{j} \leq 2^{-j} R$ for all $k \in \mathbf{N}$, so that $x_{k}^{j} \in 3 B_{j}$, and thus by $(2.1)$,

$$
\left(\frac{r_{k}^{j}}{2^{-j+2} R}\right)^{Q} \leq C_{d}^{2} \frac{\mu\left(B_{k}^{j}\right)}{\mu\left(4 B_{j}\right)} \leq C_{d}^{2} \frac{\mu\left(B_{k}^{j}\right)}{\mu\left(B_{j}\right)} \leq 2^{8} C_{d}^{3} C_{S}^{3} \delta
$$

by (3.16), so that by our choice of $\delta$,

$$
r_{k}^{j} \leq\left(2^{8} C_{d}^{3} C_{S}^{3} \delta\right)^{1 / Q} 2^{-j+2} R=\frac{2^{-j} R}{170 \lambda}
$$

Thus recalling that $F_{j} \cap B_{k}^{j} \neq \emptyset$, so that $\left(\frac{5}{4} B_{j} \backslash \frac{3}{4} B_{j+1}\right) \cap B_{k}^{j} \neq \emptyset$, we conclude that $20 \lambda B_{k}^{j} \subset B_{j-1} \backslash B_{j+2}$ (let $B_{-1}:=B(x, 2 R)$ ). Now, define Lipschitz functions

$$
\xi_{k}^{j}:=\max \left\{0,1-\frac{\operatorname{dist}\left(\cdot, 10 \lambda B_{k}^{j}\right)}{10 \lambda r_{k}^{j}}\right\}, \quad k \in \mathbf{N},
$$

so that $\xi_{k}^{j}=1$ on $10 \lambda B_{k}^{j}$ and $\xi_{k}^{j}=0$ on $X \backslash 20 \lambda B_{k}^{j}$. Using the basic properties of 1-weak upper gradients, see [3, Corollary 2.21], we obtain

$$
\int_{X} g_{\xi_{k}^{j}} d \mu \leq \frac{\mu\left(20 \lambda B_{k}^{j}\right)}{10 \lambda r_{k}^{j}}
$$


Define $V:=\bigcup_{j=0}^{\infty} \bigcup_{k=1}^{\infty} 10 \lambda B_{k}^{j}$. Now for every $i=1,2, \ldots$,

$$
\begin{aligned}
\operatorname{cap}_{1}\left(V \cap B_{i}, 4 B_{i}\right) & \leq \operatorname{cap}_{1}\left(\bigcup_{j=i-1}^{\infty} \bigcup_{k=1}^{\infty} 10 \lambda B_{k}^{j}, 4 B_{i}\right) \\
& \leq \sum_{j=i-1}^{\infty} \sum_{k=1}^{\infty} \operatorname{cap}_{1}\left(10 \lambda B_{k}^{j}, 4 B_{i}\right) \\
& \leq \sum_{j=i-1}^{\infty} \sum_{k=1}^{\infty} \int_{X} g_{\xi_{k}^{j}} d \mu \leq \sum_{j=i-1}^{\infty} \sum_{k=1}^{\infty} \frac{\mu\left(20 \lambda B_{k}^{j}\right)}{10 \lambda r_{k}^{j}} \\
& \leq C_{d}^{2} C_{B} \sum_{j=i-1}^{\infty} P\left(F_{j}, X\right) \text { by }(3.15) \\
& \leq 50 C_{d}^{2} C_{B} C_{S}^{2} \operatorname{cap}_{1}\left(W \cap B_{i-1}, 2 B_{i-1}\right) \quad \text { by }(3.9) .
\end{aligned}
$$

Thus

$$
2^{-i} R \frac{\operatorname{cap}_{1}\left(V \cap B_{i}, 4 B_{i}\right)}{\mu\left(B_{i}\right)} \leq 50 C_{d}^{3} C_{B} C_{S}^{2} 2^{-i+1} R \frac{\operatorname{cap}_{1}\left(W \cap B_{i-1}, 2 B_{i-1}\right)}{\mu\left(B_{i-1}\right)} \rightarrow 0
$$

as $i \rightarrow \infty$, since $W$ is 1 -thin at $x$. By Lemma 3.1 it is then straightforward to show that $V$ is also 1-thin at $x$. Let us also define the Lipschitz functions

$$
\eta_{k}^{j}:=\max \left\{0,1-\frac{\operatorname{dist}\left(\cdot, 5 \lambda B_{k}^{j}\right)}{5 \lambda r_{k}^{j}}\right\}, \quad j=0,1, \ldots, k=1,2, \ldots,
$$

so that $\eta_{k}^{j}=1$ on $5 \lambda B_{k}^{j}$ and $\eta_{k}^{j}=0$ on $X \backslash 10 \lambda B_{k}^{j}$, and then let

$$
\eta:=\sup _{j=0,1, \ldots, k=1,2, \ldots} \eta_{k}^{j} \text {. }
$$

Recall from Lemma 3.8 that $\bigcup_{j=0}^{\infty} F_{j} \supset W \cap B(x, R)$; thus $\eta \geq 1$ on

$$
G:=\bigcup_{j=0}^{\infty} \bigcup_{k=1}^{\infty} 5 \lambda B_{k}^{j} \supset \bigcup_{j=0}^{\infty} F_{j} \supset W \cap B(x, R) \supset A \cap B(x, R) .
$$

By [3, Lemma 1.52] we know that $g_{\eta} \leq \sum_{j=0}^{\infty} \sum_{k=1}^{\infty} g_{\eta_{k}^{j}}$. Thus for any $i=1,2, \ldots$,

$$
\begin{aligned}
\int_{B_{i}} g_{\eta} d \mu & \leq \sum_{j=0}^{\infty} \sum_{k=1}^{\infty} \int_{B_{i}} g_{\eta_{k}^{j}} d \mu \leq \sum_{j=i-1}^{\infty} \sum_{k=1}^{\infty} \int_{X} g_{\eta_{k}^{j}} d \mu \\
& \leq 50 C_{d} C_{B} C_{S}^{2} \operatorname{cap}_{1}\left(W \cap B_{i-1}, 2 B_{i-1}\right),
\end{aligned}
$$

where the last inequality follows just as in the last four lines of (3.18). Since we assumed $R \leq 1$ and so $5 \lambda r_{k}^{j} \leq 1$ by (3.17), we similarly get

$$
\|\eta\|_{L^{1}\left(B_{i}\right)} \leq 50 C_{d} C_{B} C_{S}^{2} \operatorname{cap}_{1}\left(W \cap B_{i-1}, 2 B_{i-1}\right) .
$$

Using the fact that $W$ is 1-thin at $x$ and the doubling property of $\mu$, we get (3.12). Estimating just as in the last four lines of (3.18), now with $i=1$, we get

$$
\int_{X} g_{\eta} d \mu \leq \sum_{j=0}^{\infty} \sum_{k=1}^{\infty} \int_{X} g_{\eta_{k}^{j}} d \mu \leq 50 C_{d} C_{B} C_{S}^{2} \operatorname{cap}_{1}\left(W \cap B_{0}, 2 B_{0}\right)<\infty .
$$

Thus $\eta \in N^{1,1}(X)$. Clearly $\eta=0$ on $X \backslash V$, and so $\eta \in N_{0}^{1,1}(V)$. 


\section{1-strict subsets}

In this section we study 1-strict subsets which are defined as follows.

Definition 4.1. A set $A \subset D$ is a 1-strict subset of $D \subset X$ if there is a function $u \in N_{0}^{1,1}(D)$ such that $u=1$ on $A$.

Equivalently, $A$ is a 1 -strict subset of $D$ if $\operatorname{cap}_{1}(A, D)<\infty$. In [22, Proposition 6.7] we proved the following result by using the weak Cartan property (Theorem 3.3).

Proposition 4.2. Let $U \subset X$ be 1-finely open and let $x \in U$. Then there exists a 1-finely open set $W$ such that $x \in W \subset U$, and a function $w \in \operatorname{BV}(X)$ such that $0 \leq w \leq 1$ on $X, w^{\wedge}=1$ on $W$, and $\operatorname{spt} w \Subset U$.

This kind of formulation is sufficient for some purposes, but now we are able to improve it by replacing $w \in \mathrm{BV}(X)$ with $w \in N^{1,1}(X)$. The following is our main result on the existence of 1 -strict subsets.

Theorem 4.3. Let $U \subset X$ be 1-finely open and let $x \in U$. Then there exists a 1-finely open set $W$ such that $x \in W \subset U$, and a function $w \in N_{0}^{1,1}(U)$ such that $0 \leq w \leq 1$ on $X, w=1$ on $W$, and spt $w \Subset U$. Moreover, if $\operatorname{Cap}_{1}(\{x\})=0$, then $\|w\|_{N^{1,1}(X)}$ can be made arbitrarily small.

Proof. Applying Theorem 3.11 with the choice $A=X \backslash U$, we find a number $R>0$, open sets $G \subset V \subset X$, and a function $\eta \in N_{0}^{1,1}(V)$ such that $B(x, R) \subset G \cup U$, $V$ is 1 -thin at $x, 0 \leq \eta \leq 1$ on $X, \eta=1$ on $G$, and

$$
\lim _{r \rightarrow 0} \frac{r}{\mu(B(x, r))}\|\eta\|_{N^{1,1}(B(x, r))}=0 .
$$

Choose $0<r \leq R$ such that $r\|\eta\|_{N^{1,1}(B(x, r))} / \mu(B(x, r)) \leq 1$ and let

$$
\rho:=\max \left\{0,1-\frac{4 \operatorname{dist}(\cdot, B(x, r / 2))}{r}\right\} \in \operatorname{Lip}(X),
$$

so that $0 \leq \rho \leq 1$ on $X, \rho=1$ on $B(x, r / 2)$, and spt $\rho \Subset B(x, r)$. Then let $w:=(1-\eta) \rho$. By the Leibniz rule (see [3, Theorem 2.15]), we have $w \in N^{1,1}(X)$ and

$$
\int_{X} g_{w} d \mu=\int_{B(x, r)} g_{w} d \mu \leq \int_{B(x, r)} g_{\eta} d \mu+\int_{B(x, r)} g_{\rho} d \mu \leq \frac{\mu(B(x, r))}{r}+\frac{4 \mu(B(x, r))}{r} .
$$

Thus $\|w\|_{N^{1,1}(X)} \leq(5 / r+1) \mu(B(x, r))$. If $\operatorname{Cap}_{1}(\{x\})=0$, then also $\mathcal{H}(\{x\})=0$ by (2.4), and so we can make $\mu(B(x, r)) / r$ as small as we like by choosing suitable $r$. Then we can also make $\|w\|_{N^{1,1}(X)}$ arbitrarily small.

Regardless of the value of $\operatorname{Cap}_{1}(\{x\})$, the set $V$ is 1 -thin at $x$, that is, $x \notin b_{1} V$. Since $V$ is open we have $V \subset b_{1} V$; recall (2.10) and the comment after it. We know that $\bar{V}^{1}=V \cup b_{1} V$ by [19, Corollary 3.5], so in conclusion $x \notin \bar{V}^{1}$. Thus

$$
W:=B(x, r / 2) \backslash \bar{V}^{1} \subset\{w=1\}
$$

is a 1-finely open neighborhood of $x$. Finally, spt $w$ is compact and

$$
\text { spt } w \subset \operatorname{spt} \rho \backslash G \subset(U \cup G) \backslash G \subset U,
$$

so that spt $w \Subset U$. Clearly now $w \in N_{0}^{1,1}(U)$.

Let us make a few more observations concerning 1-strict subsets. In general it is not clear which subsets $A$ of a set $D$ are 1-strict subsets. If $A$ is a compact subset of 
an open set $D$, we obviously have $\operatorname{cap}_{1}(A, D)<\infty$, and the test function can even be chosen to be Lipschitz. When $A$ is a compact subset of a 1-quasiopen set $D$, we cannot necessarily choose a Lipschitz test function but one might nonetheless suspect that $\operatorname{cap}_{1}(A, D)<\infty$. However, this is not always the case.

Example 4.4. Let $X=\mathbf{R}^{2}$ (unweighted), denote the origin by 0 , and let

$$
A:=\bigcup_{j=1}^{\infty} A_{j} \cup\{0\} \quad \text { with } \quad A_{j}:=\left\{2^{-j}\right\} \times[-1 /(2 j), 1 /(2 j)] .
$$

Denoting $A_{j}^{\varepsilon}:=\left\{x \in X: \operatorname{dist}\left(x, A_{j}\right)<\varepsilon\right\}$, with $\varepsilon>0$, let

$$
D:=\bigcup_{j=1}^{\infty} D_{j} \cup\{0\} \quad \text { with } \quad D_{j}:=A_{j}^{2^{-3 j}} .
$$

Since all the sets $D_{j}$ are disjoint, it is straightforward to check that

$$
\operatorname{cap}_{1}(A, D)=\sum_{j=1}^{\infty} \operatorname{cap}_{1}\left(A_{j}, D_{j}\right)=\sum_{j=1}^{\infty} \frac{1}{j}=\infty .
$$

Now $A$ is clearly a compact set, and $D$ is 1-quasiopen since $D \cup B(0, r)$ is an open set for every $r>0$.

One can also make the sets $A, D$ connected by adding the line $(0,1 / 2] \times\{0\}$ to $A$, and by adding e.g. the sets $\left(2^{-j-1}, 2^{-j}\right) \times\left(-2^{-j-1}, 2^{-j-1}\right)$ to $D$; then we still have $\operatorname{cap}_{1}(A, D)=\infty$ but the calculation is somewhat more complicated.

The variational 1-capacity is an outer capacity in the following weak sense.

Proposition 4.5. Let $A \subset D \subset X$. Then

$$
\operatorname{cap}_{1}(A, D)=\inf _{\substack{V-\text { quasiopen } \\ A \subset V \subset D}} \operatorname{cap}_{1}(V, D) .
$$

Proof. We can assume that $\operatorname{cap}_{1}(A, D)<\infty$. Fix $0<\varepsilon<1$. Take $u \in N_{0}^{1,1}(D)$ such that $u=1$ on $A$ and $\int_{X} g_{u} d \mu<\operatorname{cap}_{1}(A, D)+\varepsilon$. The set $V:=\{u>1-\varepsilon\}$ is 1-quasiopen by Theorem 2.5, and

$$
\operatorname{cap}_{1}(V, D) \leq \int_{X} g_{u /(1-\varepsilon)} d \mu=\frac{\int_{X} g_{u} d \mu}{1-\varepsilon} \leq \frac{\operatorname{cap}_{1}(A, D)+\varepsilon}{1-\varepsilon} .
$$

Since $0<\varepsilon<1$ was arbitrary, we have the result.

Even though 1-quasiopen sets and 1-finely open sets are very closely related (recall Theorem 2.12), it is not clear whether the following holds.

Open Problem. If $D \subset X$ and $A \subset$ fine-int $D$, do we have

$$
\operatorname{cap}_{1}(A, D)=\inf _{\substack{V \text { 1-inely open } \\ A \subset V \subset D}} \operatorname{cap}_{1}(V, D) ?
$$

Note that according to Theorem 4.3, the above property does hold in the very special case when $A$ is a point with 1-capacity zero.

Let us say that a set $K \subset X$ is 1-quasiclosed if $X \backslash K$ is 1-quasiopen. Now we can show that 1-strict subsets have the following continuity.

Proposition 4.6. Let $D \subset X$ and let $K_{1} \supset K_{2} \supset \ldots$ be bounded 1-quasiclosed subsets of $D$ such that $\operatorname{cap}_{1}\left(K_{1}, D\right)<\infty$. Then for $K:=\bigcap_{i=1}^{\infty} K_{i}$ we have

$$
\operatorname{cap}_{1}(K, D)=\lim _{i \rightarrow \infty} \operatorname{cap}_{1}\left(K_{i}, D\right) \text {. }
$$


We will show in Example 4.7 below that the assumption $\operatorname{cap}_{1}\left(K_{1}, D\right)<\infty$ is needed.

Proof. Fix $\varepsilon>0$. By Proposition 4.5 we find a 1-quasiopen set $V$ such that $K \subset V \subset D$ and $\operatorname{cap}_{1}(V, D)<\operatorname{cap}_{1}(K, D)+\varepsilon$. For each $j \in \mathbf{N}$ we find an open set $\widetilde{G}_{j} \subset X$ such that $V \cup \widetilde{G}_{j}$ is open and $\operatorname{Cap}_{1}\left(\widetilde{G}_{j}\right) \rightarrow 0$ as $j \rightarrow \infty$. For each $i, j \in \mathbf{N}$, we find an open set $G_{i, j} \subset X$ such that $K_{i} \backslash G_{i, j}$ is compact and $\operatorname{Cap}_{1}\left(G_{i, j}\right)<2^{-i-j}$. Letting $G_{j}:=\widetilde{G}_{j} \cup \bigcup_{i=1}^{\infty} G_{i, j}$ for each $j \in \mathbf{N}$, we have that each $V \cup G_{j}$ is open, each $K_{i} \backslash G_{j}$ is compact, and $\operatorname{Cap}_{1}\left(G_{j}\right) \rightarrow 0$ as $j \rightarrow \infty$. Then for each $j \in \mathbf{N}$ we find a function $w_{j} \in N^{1,1}(X)$ such that $0 \leq w_{j} \leq 1$ on $X, w_{j}=1$ on $G_{j}$, and $\left\|w_{j}\right\|_{N^{1,1}(X)} \rightarrow 0$ as $j \rightarrow \infty$. Passing to a subsequence (not relabeled), we can assume that $w_{j} \rightarrow 0$ a.e.

Since $\operatorname{cap}_{1}\left(K_{1}, D\right)<\infty$, we find $v \in N_{0}^{1,1}(D)$ such that $0 \leq v \leq 1$ on $X$ and $v=1$ on $K_{1}$. For each $j \in \mathbf{N}$, let $\rho_{j}:=v w_{j}$. Then $\left\|\rho_{j}\right\|_{L^{1}(X)} \rightarrow 0$ as $j \rightarrow \infty$, and by the Leibniz rule (see [3, Theorem 2.15]),

$$
\int_{X} g_{\rho_{j}} d \mu \leq \int_{X} g_{w_{j}} d \mu+\int_{X} w_{j} g_{v} d \mu \rightarrow 0
$$

as $j \rightarrow \infty$; for the second term this follows from Lebesgue's dominated convergence theorem. Thus $\operatorname{cap}_{1}\left(G_{j} \cap K_{1}, D\right) \rightarrow 0$. Fix $j \in \mathbf{N}$ such that $\operatorname{cap}_{1}\left(G_{j} \cap K_{1}, D\right)<\varepsilon$. Since every $K_{i} \backslash G_{j}$ is compact and $V \cup G_{j}$ is open, for some $i \in \mathbf{N}$ we have $K_{i} \backslash G_{j} \subset$ $V \cup G_{j}$. Thus $K_{i} \subset V \cup G_{j}$. Then

$$
\begin{aligned}
\operatorname{cap}_{1}\left(K_{i}, D\right) & \leq \operatorname{cap}_{1}\left(V \cup\left(G_{j} \cap K_{1}\right), D\right) \leq \operatorname{cap}_{1}(V, D)+\operatorname{cap}_{1}\left(G_{j} \cap K_{1}, D\right) \\
& \leq \operatorname{cap}_{1}(K, D)+\varepsilon+\operatorname{cap}_{1}\left(G_{j} \cap K_{1}, D\right) \leq \operatorname{cap}_{1}(K, D)+2 \varepsilon .
\end{aligned}
$$

Since $\varepsilon>0$ was arbitrary, the proof is concluded.

Example 4.7. In the notation of Example 4.4, let $K_{i}:=\bigcup_{j=i}^{\infty} A_{j} \cup\{0\}$ for each $i \in \mathbf{N}$. These are compact sets and similarly as in Example 4.4 we find that $\operatorname{cap}_{1}\left(K_{i}, D\right)=\infty$ for every $i \in \mathbf{N}$. However, $\operatorname{cap}_{1}(K, D)=0$ for $K:=\bigcap_{i=1}^{\infty} K_{i}=\{0\}$, by the fact that a point has 1 -capacity zero and by using $(2.3)$.

\section{Application to fine Sobolev spaces}

Björn-Björn-Latvala [7] have studied different definitions of Newton-Sobolev spaces on quasiopen sets in metric spaces in the case $1<p<\infty$. As an application of the theory we have developed, we show that the analogous results hold for $p=1$.

First we prove the following fact in a very similar way as it is proved in the case $1<p<\infty$, see [8, Theorem 1.4(b)] and [6, Theorem 4.9(b)]. Recall that a function $u$ defined on a set $U \subset X$ is 1-quasicontinuous on $U$ if for every $\varepsilon>0$ there is an open set $G \subset X$ such that $\operatorname{Cap}_{1}(G)<\varepsilon$ and $\left.u\right|_{U \backslash G}$ is continuous (as a real-valued function).

Theorem 5.1. A function $u$ on a 1-quasiopen set $U$ is 1-quasicontinuous on $U$ if and only if it is finite 1-q.e. and 1-finely continuous 1-q.e. on $U$.

Proof. To prove one direction, suppose there is a set $N \subset U$ such that $\operatorname{Cap}_{1}(N)=$ 0 and $u$ is finite and 1-finely continuous at every point in $V:=U \backslash N$. By Theorem 2.12, we can assume that $V$ is 1-finely open. Let $\left\{\left(a_{j}, b_{j}\right)\right\}_{j=1}^{\infty}$ be an enumeration of all intervals in $\mathbf{R}$ with rational endpoints and let

$$
V_{j}:=\left\{x \in V: a_{j}<u(x)<b_{j}\right\} .
$$


By the 1-fine continuity of $u$, the sets $V_{j}$ are 1-finely open. Hence by Theorem 2.12, they are also 1-quasiopen. Fix $\varepsilon>0$. There are open sets $G_{j} \subset X$ such that $\operatorname{Cap}_{1}\left(G_{j}\right)<2^{-j-1} \varepsilon$ and each $V_{j} \cup G_{j}$ is open. Since Cap Cas $_{1}$ an outer capacity, there is also an open set $G_{N} \supset N$ such that $\operatorname{Cap}_{1}\left(G_{N}\right)<\varepsilon / 2$. Now

$$
G:=G_{N} \cup \bigcup_{j=1}^{\infty} G_{j}
$$

is an open set such that $\operatorname{Cap}_{1}(G)<\varepsilon$, and $\left.u\right|_{U \backslash G}$ is continuous since $V_{j} \cup G$ are open sets.

To prove the converse direction, by Theorem 2.12 we know that $U=V \cup N$, where $V$ is 1-finely open and $\mathcal{H}(N)=0$, and then also $\operatorname{Cap}_{1}(N)=0$ by (2.4). By the quasicontinuity of $u$, for each $j \in \mathbf{N}$ we find an open set $G_{j} \subset X$ such that $\operatorname{Cap}_{1}\left(G_{j}\right)<1 / j$ and $\left.u\right|_{V \backslash G_{j}}$ is continuous. By (2.11), we have $\operatorname{Cap}_{1}\left({\overline{G_{j}}}^{1}\right)=\operatorname{Cap}_{1}\left(G_{j}\right)$ for each $j \in \mathbf{N}$, and so the set

$$
A:=N \cup \bigcap_{j=1}^{\infty}{\overline{G_{j}}}^{1}
$$

satisfies $\operatorname{Cap}_{1}(A)=0$. If $x \in U \backslash A$, then $x \in V \backslash{\overline{G_{j}}}^{-1}$ for some $j \in \mathbf{N}$. Since $V \backslash{\overline{G_{j}}}^{1}$ is a 1-finely open set and $\left.u\right|_{V \backslash \bar{G}_{j}}$ is continuous, it follows that $u$ is finite and 1-finely continuous at $x$.

We will need the following quasi-Lindelöf principle from [22].

Theorem 5.2. [22, Theorem 5.2] For every family $\mathcal{V}$ of 1-finely open sets there is a countable subfamily $\mathcal{V}^{\prime}$ such that

$$
\operatorname{Cap}_{1}\left(\bigcup_{V \in \mathcal{V}} V \backslash \bigcup_{V^{\prime} \in \mathcal{V}^{\prime}} V^{\prime}\right)=0
$$

From now on, $U \subset X$ is always a 1-quasiopen set. Note that 1-quasiopen sets are $\mu$-measurable by [5, Lemma 9.3].

Definition 5.3. A family $\mathcal{B}$ of 1-quasiopen sets is a 1-quasicovering of $U$ if it is countable, $\bigcup_{V \in \mathcal{B}} V \subset U$, and $\operatorname{Cap}_{1}\left(U \backslash \bigcup_{V \in \mathcal{B}} V\right)=0$. If every $V \in \mathcal{B}$ is a 1finely open 1-strict subset of $U$ with $V \Subset U$, then $\mathcal{B}$ is a 1-strict quasicovering of $U$. Moreover, we say that

1. $u \in N_{\text {fine-loc }}^{1,1}(U)$ if $u \in N^{1,1}(V)$ for every 1-finely open 1-strict subset $V \Subset U$;

2. $u \in N_{\text {quasi-loc }}^{1,1}(U)$ (respectively $L_{\text {quasi-loc }}^{1}(U)$ ) if there is a 1-quasicovering $\mathcal{B}$ of $U$ such that $u \in N^{1,1}(V)$ (respectively $L^{1}(V)$ ) for every $V \in \mathcal{B}$.

Proposition 5.4. There exists a 1-strict quasicovering $\mathcal{B}$ of $U$. Moreover, the associated Newton-Sobolev functions can be chosen compactly supported in $U$.

Proof. By Theorem 2.12, we have $U=V \cup N$, where $V$ is 1-finely open and $\mathcal{H}(N)=0$, and then also $\operatorname{Cap}_{1}(N)=0$ by (2.4). For every $x \in V$, by Theorem 4.3 we find a 1-finely open set $V_{x} \ni x$ such that $V_{x} \Subset V$ and an associated function $v_{x} \in N_{0}^{1,1}(V)$ such that $0 \leq v_{x} \leq 1$ on $X, v_{x}=1$ on $V_{x}$, and $\operatorname{spt} v_{x} \Subset V$. The collection $\mathcal{B}^{\prime}:=\left\{V_{x}\right\}_{x \in V}$ covers $V$, and by the quasi-Lindelöf principle (Theorem 5.2) and the fact that $\operatorname{Cap}_{1}(U \backslash V)=0$, there exists a countable subcollection $\mathcal{B} \subset \mathcal{B}^{\prime}$ such that $\operatorname{Cap}_{1}\left(U \backslash \bigcup_{V_{x} \in \mathcal{B}} V_{x}\right)=0$. 
It follows that $N_{\text {fine-loc }}^{1,1}(U) \subset N_{\text {quasi-loc }}^{1,1}(U)$. From now on, since the proofs given in [7] in the case $1<p<\infty$ apply almost verbatim also in our setting, we will only point out the differences with [7].

Theorem 5.5. Let $u \in N_{\text {quasi-loc }}^{1,1}(U)$. Then $u$ if finite 1-q.e. and 1-finely continuous 1-q.e. on $U$. Thus $u$ is also 1-quasicontinuous on $U$.

Proof. Follow verbatim the proof of [7, Theorem 4.4], except that replace the reference to [7, Proposition 4.2] by Proposition 5.4, and the references to [6, Theorem $4.9(\mathrm{~b})]$ and $[8$, Theorem $1.4(\mathrm{~b})]$ by Theorem 5.1 .

Definition 5.6. A nonnegative function $\widetilde{g}_{u}$ on $U$ is a 1-fine upper gradient of $u \in N_{\text {quasi-loc }}^{1,1}(U)$ if there is a quasicovering $\mathcal{B}$ of $U$ such that for every $V \in \mathcal{B}$, $u \in N^{1,1}(V)$ and $\widetilde{g}_{u}=g_{u, V}$ a.e. on $V$, where $g_{u, V}$ is the minimal 1-weak upper gradient of $u$ in $V$.

Lemma 5.7. If $u \in N_{\text {quasi-loc }}^{1,1}(U)$, then it has a unique (in the a.e. sense) 1-fine upper gradient $\widetilde{g}_{u}$.

Proof. Follow verbatim the proof of [7, Lemma 5.2].

Theorem 5.8. If $u \in N_{\text {quasi-loc }}^{1,1}(U)$ and $\widetilde{g}_{u}$ is a 1-fine upper gradient of $u$, then $\widetilde{g}_{u}$ is a 1-weak upper gradient of $u$ in $U$.

Proof. Follow verbatim the proof of [7, Theorem 5.3].

Proposition 5.9. If $u \in N_{\text {quasi-loc }}^{1,1}(U)$, then there is a 1-strict quasicovering $\mathcal{B}$ of $U$ such that for every $V \in \mathcal{B}$, there exists $u_{V} \in N^{1,1}(X)$ with $u=u_{V}$ on $V$.

Proof. Follow verbatim the proof of [7, Proposition 5.5], except that replace the reference to [7, Theorem 4.4] by Theorem 5.5, and [7, Proposition 4.2] by Proposition 5.4.

The following definition is originally from Kilpeläinen-Malý [17].

Definition 5.10. Let $U \subset \mathbf{R}^{n}$. A function $u \in L^{1}(U)$ is in $W^{1,1}(U)$ if

1. there is a quasicovering $\mathcal{B}$ of $U$ such that for every $V \in \mathcal{B}$ there is an open set $G_{V} \supset V$ and $u_{V} \in W^{1,1}\left(G_{V}\right)$ such that $u=u_{V}$ on $V$, and

2. the fine gradient $\nabla u$, defined by $\nabla u=\nabla u_{V}$ a.e. on each $V \in \mathcal{B}$, also belongs to $L^{1}(U)$.

Moreover, let

$$
\|u\|_{W^{1,1}(U)}:=\int_{U}(|u|+|\nabla u|) d x
$$

Recall that we constantly assume $U$ to be a 1-quasiopen set.

Theorem 5.11. Let $U \subset \mathbf{R}^{n}$. Then $u \in W^{1,1}(U)$ if and only if there exists $v \in N^{1,1}(U)$ such that $v=u$ a.e. on $U$. Moreover, $g_{v}=|\nabla u|$ a.e. on $U$ and $\|v\|_{N^{1,1}(U)}=\|u\|_{W^{1,1}(U)}$.

Here $g_{v}$ is the minimal 1-weak upper gradient of $v$ in $U$.

Proof. Follow verbatim the proof of [7, Theorem 5.7], except that replace the reference to [7, Proposition 5.5] by Proposition 5.9, [3, Proposition A.12] by [3, Corollary A.4], and [7, Theorem 5.4] by Theorem 5.8. 
Returning momentarily to the metric space setting, define the space

$$
\widehat{N}^{1,1}(U):=\left\{u: u=v \text { a.e. for some } v \in N^{1,1}(U)\right\} .
$$

Theorem 5.12. Let $u \in \widehat{N}^{1,1}(U)$. Then $u \in N^{1,1}(U)$ if and only if $u$ is 1 quasicontinuous on $U$.

Proof. Assume that $u$ is 1-quasicontinuous on $U$. There is $v \in N^{1,1}(U)$ such that $u=v$ a.e. on $U$. By Theorem 5.5, $v$ is 1-quasicontinuous on $U$. By [3, Proposition 5.23] and [9, Proposition 4.2], $u=v$ 1-q.e. on $U$, and thus $u \in N^{1,1}(U)$ by (2.3).

The converse follows from Theorem 5.5.

Theorem 5.13. Let $U \subset \mathbf{R}^{n}$, and let $u$ be an everywhere defined function on $U$. Then $u \in N^{1,1}(U)$ if and only if $u \in W^{1,1}(U)$ and $u$ is 1-quasicontinuous. Moreover, then $\|u\|_{N^{1,1}(U)}=\|u\|_{W^{1,1}(U)}$.

Proof. This follows from Theorems 5.11 and 5.12.

\section{References}

[1] Adams, D., and L. I. Hedberg: Function spaces and potential theory. - Grundlehren Math. Wiss. 314, Springer-Verlag, Berlin, 1996.

[2] Ambrosio, L., N. Fusco, and D. Pallara: Functions of bounded variation and free discontinuity problems. - Oxford Math. Monogr., The Clarendon Press, Oxford Univ. Press, New York, 2000.

[3] BJöRn, A., and J. BJöRN: Nonlinear potential theory on metric spaces. - EMS Tracts Math. 17, Eur. Math. Soc. (EMS), Zürich, 2011.

[4] BJÖRn, A., and J. BJöRn: The variational capacity with respect to nonopen sets in metric spaces. - Potential Anal. 40:1, 2014, 57-80.

[5] BJÖRn, A., and J. BJÖRN: Obstacle and Dirichlet problems on arbitrary nonopen sets in metric spaces, and fine topology. - Rev. Mat. Iberoam. 31:1, 2015, 161-214.

[6] BJöRn, A., J. BJöRn, and V. Latvala: The weak Cartan property for the p-fine topology on metric spaces. - Indiana Univ. Math. J. 64:3, 2015, 915-941.

[7] BJörn, A., J. Buörn, and V. Latvala: Sobolev spaces, fine gradients and quasicontinuity on quasiopen sets. - Ann. Acad. Sci. Fenn. Math. 41:2, 2016, 551-560.

[8] BJörn, A., J. BJöRn, and V. Latvala: The Cartan, Choquet and Kellogg properties for the fine topology on metric spaces. - J. Anal. Math. (to appear).

[9] BJÖRN, A., J. BJÖRN, and J. MALÝ: Quasiopen and p-path open sets, and characterizations of quasicontinuity. - Potential Anal. 46:1, 2017, 181-199.

[10] BJörn, A., J. BJörn, and N. Shanmugalingam: Quasicontinuity of Newton-Sobolev functions and density of Lipschitz functions on metric spaces. - Houston J. Math. 34:4, 2008, $1197-1211$.

[11] Evans, L. C., and R.F. Gariepy: Measure theory and fine properties of functions. Stud. Adv. Math., CRC Press, Boca Raton, 1992.

[12] Federer, H.: Geometric measure theory. - Grundlehren Math. Wiss. 153, Springer-Verlag New York Inc., New York, 1969.

[13] Giusti, E.: Minimal surfaces and functions of bounded variation. - Monographs in Mathematics 80, Birkhäuser Verlag, Basel, 1984.

[14] Hakkarainen, H., and J. Kinnunen: The BV-capacity in metric spaces. - Manuscripta Math. 132:1-2, 2010, 51-73. 
[15] Heinonen, J., T. Kilpeläinen, and O. Martio: Nonlinear potential theory of degenerate elliptic equations. - Dover Publications, Inc., Mineola, NY, 2006.

[16] Heinonen, J., and P. Koskela: Quasiconformal maps in metric spaces with controlled geometry. - Acta Math. 181:1, 1998, 1-61.

[17] Kilpeläinen, T., and J. MalÝ: Supersolutions to degenerate elliptic equation on quasi open sets. - Comm. Partial Differential Equations 17:3-4, 1992, 371-405.

[18] Kinnunen, J., R. Korte, N. Shanmugalingam, and H. Tuominen: Lebesgue points and capacities via the boxing inequality in metric spaces. - Indiana Univ. Math. J. 57:1, 2008, 401-430.

[19] Lahti, P.: A Federer-style characterization of sets of finite perimeter on metric spaces. - Calc. Var. Partial Differential Equations 56:5, 2017, 56:150.

[20] LAhti, P.: A notion of fine continuity for BV functions on metric spaces. - Potential Anal. 46:2, 2017, 279-294.

[21] Lahti, P.: Superminimizers and a weak Cartan property for $p=1$ in metric spaces. - J. Anal. Math. (to appear).

[22] Lahti, P.: The Choquet and Kellogg properties for the fine topology when $p=1$ in metric spaces. - Preprint https://arxiv.org/abs/1712.08027, 2017.

[23] MaLÝ, J., and W. Ziemer: Fine regularity of solutions of elliptic partial differential equations. - Math. Surveys Monogr. 51, Amer. Math. Soc., Providence, RI, 1997.

[24] Miranda, M. JR.: Functions of bounded variation on "good" metric spaces. - J. Math. Pures Appl. (9) 82:8, 2003, 975-1004.

[25] Shanmugalingam, N.: Newtonian spaces: An extension of Sobolev spaces to metric measure spaces. - Rev. Mat. Iberoamericana 16:2, 2000, 243-279.

[26] Ziemer, W.P.: Weakly differentiable functions. Sobolev spaces and functions of bounded variation. - Grad. Texts in Math. 120. Springer-Verlag, New York, 1989. 